\title{
APURDUTM
}

Euskal ikerketen aldizkaria | Revue d'études basques |

Revista de estudios vascos | Basque studies review

$1 \mid 1996$

Numéro I

\section{À propos du système pronominal dans Guero de P. de Axular (1643)}

\section{Georges Rebuschi}

\section{OpenEdition}

\section{Journals}

Édition électronique

URL : http://journals.openedition.org/lapurdum/1864

DOI : 10.4000/lapurdum. 1864

ISSN : 1965-0655

Éditeur

IKER

Édition imprimée

Date de publication : 1 octobre 1996

Pagination : 73-85

ISBN : 2-84127-106-4

ISSN : $1273-3830$

Référence électronique

Georges Rebuschi, «À propos du système pronominal dans Guero de P. de Axular (1643) », Lapurdum [En ligne], 1 | 1996, mis en ligne le 01 septembre 2010, consulté le 30 janvier 2020. URL : http:// journals.openedition.org/lapurdum/1864; DOI : 10.4000/lapurdum.1864 
Georges REBUSCHI

\section{À PROPOS DU SYSTÈME PRONOMINAL DANS GUERO DE P. DE AXULAR (1643)}

1. Dans WSGP (Rebuschi, 1995)*, j'ai tenté de dégager les principes grammaticaux régissant la distribution complémentaire des pronoms génitifs (dorénavant $P G$ ), respectivement faibles et forts (1), en basque classique du Nord (tant labourdin que central : bas-navarrais et oriental : souletin) - pronoms dont je rappelle les formes en (1) ci-après ainsi que de décrire l'évolution de ce système, essentiellement jusqu'au $\mathrm{XIX}^{\mathrm{e}}$ siècle.

(1) Formes faibles et fortes des pronoms génitifs en labourdin classique

\begin{tabular}{|c|c|c|c|c|c|c|c|}
\hline & $1 \mathrm{sg}$ & $2 \mathrm{sg}$ & $3 \mathrm{sg}$ & $1 \mathrm{pl}$ & $2 \mathrm{pol}$ & $2 \mathrm{pl}$ & $3 \mathrm{pl}$ \\
\hline Génitifs faibles & ene & hire & haren & gure & zure & zuen & haien \\
\hline Génitifs forts & neure & heure & bere & geure & zeure & zeuen & bere \\
\hline
\end{tabular}

Faute de place, il ne m'a pas été possible d'inclure trois appendices qui accompagnaient originellement le manuscrit : l'un concernait les sujets pronominaux génitifs, comme dans les extraits de Detchepare (1545) cités en $(2 \mathrm{a}, \mathrm{b})$; le second, la question des arguments obliques et circonstants chez Liçarrague (1571), cf. (3); et le dernier, les contreexemples (apparents ou réels) trouvés dans l'édition de 1964 de $G(u)$ ero par L. Villasante.

(2) a. ENE gaizki penatzeko hain ederrik sortu zen.

[Detchepare, 1545, 144:9]

'C'est pour me faire cruellement souffrir qu'elle est née si belle'.

b. NEURE gaizki penatzeko har zintzadan amore.

[Detchepare, 1545, 156:29] ${ }^{(2)}$

'C'est pour m'infliger des peines cruelles que je m'épris de vous.' ${ }^{(3)}$

* Je souhaite remercier R. L. Trask pour ses commentaires sur le texte de 1995, et J.-B. Orpustan pour ses remarques sur une première version du précédent et de celui-ci : ils m'ont tous deux permis de préciser davantage quelques points. Mais je reste évidemment responsable des erreurs d'analyse qu'on pourrait trouver ici. 
(3) Mt 16,8

'Pourquoi faites-vous en vous-mêmes cette réflexion que vous n'avez pas de pain ?'

Zer dihardukatZUE pro $_{\mathrm{i}}$ ZEURON $_{\mathrm{i}}$ artean, zeren ogirik hartu eztuzuen?

[Liçarrague, 1571]

Zergatik darasaZUE pro $_{\mathrm{i}}$ ZEUEN $_{\mathrm{i}}$ baitan eztuZUEla hartu ogirik ? [Haraneder, 1742]

Zer derabilkaZUE pro $_{\mathrm{i}}$ ZUEN $_{\mathrm{i}}$ artean, ez duzuelakoz ogirik hartu ? [Duvoisin, 1859-65] (f)

Une publication indépendante, pour les deux premiers appendices mentionnés à l'instant, est en cours d'élaboration. Dans les pages qui suivent, c'est le contenu du troisième appendice qui m'intéressera. Mais pour en comprendre l'enjeu, il faut d'abord résumer les acquis essentiels de WSGP, en particulier en ce qui concerne le labourdin.

2. Si l'on se fie précisément à la distribution des deux types de PG, on peut définir le labourdin classique stricto sensu comme la forme de basque écrite sur la côte basque française dans la première moitié du XVII' siècle, et illustrée en particulier par Etcheverry de Ciboure (1627) et Axular (1643) ${ }^{(5)}$. J'ai choisi d'appeler les deux séries de PG respectivement "faible" et "forte" pour des raisons essentiellement phonologiques (présence d'une voyelle diphtonguée dans la série "forte", aux deux premières personnes du moins, vs. absence générale de toute diphtongaison dans la série "faible"), et afin d'éviter tout a priori concernant leur valeur grammaticale ou sémantico-énonciative (on va de plus voir que cette dernière valeur est absolument nulle; cf. aussi la note 1).

Le génitif signalant ou bien la relation de "possession" au sens large, ou bien la rection par défaut du complément d'une postposition ${ }^{(0)}$, on peut décrire la distribution des PG faibles et forts comme suit :

(4) Quand un PG contenu dans un syntagme nominal (SN) ou un syntagme postpositionnel (SP) coréfère à un argument du verbe dont ce SN ou ce SP dénote un actant ou un circonstant, la forme forte est obligatoire.

Dans les autres cas de figure, seule la forme faible est licite.

(J'appelle "arguments d'un verbe" les SN à l'un des trois cas qui entraînent l'apparition d'une marque d'accord sur le verbe conjugué, auxiliaire ou lexical : absolutif, ergatif ou datif, si la proposition est fléchie; ces SN ont le même statut d'arguments lorsque la proposition n'est pas conjuguée.)

3. Voici quelques illustrations de ces propriétés, et de quelques autres, concernant l'emploi des formes fortes. ${ }^{(7)}$ 
3.1. L'ordre des mots et syntagmes est non pertinent : le SN possessivé peut précéder l'antécédent, cf. (5), même dans le cas où le génitif est dans le SN sujet, cf. (6), ou encore si cet antécédent est indéfini, cf. (7) ${ }^{(8)}$ :

(5) NEURE $E_{j}$ onak neuk $\mathrm{k}_{\mathrm{i}}$ nahi ditut gozatu. [Ax, 157/317]

'(De) MES biens je veux jouir moi-même.'

(6) $\mathrm{BERE}_{\mathrm{i}}$ anaiak Josephi $\mathrm{i}_{\mathrm{i}}$ mintzatu zeitzanean... [Ax, 218/432]

'Quand SES frères parlèrent à Joseph...'

(7) BERE $_{i}$ gorputzeko gaitzak emaiten baitio bat-bederari $i_{i}$ egitekorik asko. [Ax, 137/279]

lit. 'Parce que la douleur de SON corps donne à chacun suffisamment de quoi faire.'

3.2. Le même possessif ou génitif fort apparaît dans l'expression [...burua] '(ma/ta/sa) tête'qui s'interprète sémantiquement comme un argument réfléchi - mais syntaxiquement, c'est un SN de $3^{\mathrm{e}}$ personne, comme le montre l'accord en $3 \mathrm{sg}$, quelle que soit la personne du référent, et le fait que cette expression puisse être coordonnée à une autre (l'antécédent n'a pas besoin d'être explicite ; s'il est implicite, je le représente par pro):

(8) Zeren orduan pro $_{\mathrm{i}}$-ERG ZEURE $\mathrm{j}_{\mathrm{j}}$ etsaia, ZEURE BURUA eta deabrua ere garaitzen baitituTZU. [Ax, 212/420]

'Parce qu'alors vous dominez votre ennemi, vous-même et même le diable.'

3.3. Lorsqu'une proposition est nominalisée, rien ne change, tous les arguments, sujet inclus, pouvant être explicites ou implicites (je note cependant le sujet sous-entendu autrement, par $P R O$, dans la mesure où, contrairement au "petit pro", il peut avoir une valeur indéfinie ou générique).

- Cas avec coréférents explicites :

(9) Etzatekeien hobe [obra on horion guztion ZERORREK ZE $_{\mathrm{i}} \ldots$ ZEURE $_{\mathrm{j}}$ eskuz egitea] ? [Ax, 157/317]

'N'aurait-il pas été meilleur que vous fassiez [lit. : vous-ERG faire] toutes ces bonnes œuvres vous-même, de votre (propre) main?'

Ici, le PG de 2 e p. pol. est contenu dans un syntagme adjoint ou adverbial qui se rapporte au verbe non-fléchi egitea; comme ce verbe a un sujet, l'emphatique zerorrek, également de $2^{\mathrm{c}} \mathrm{p}$. polie, la forme forte est obligatoire. Le même raisonnement s'applique au passage suivant, où la personne en cause est la première du pluriel : 
(10) pro $_{\mathrm{i}}$-ERG iustiziaz eztu behin ere usatzen, $\left[\mathrm{GUK}_{\mathrm{j}}\right.$ lehenik GEURE $\mathrm{E}_{\mathrm{j}}$ bekatuez pro $_{\mathrm{i}}$ hartara behartu gabe]. [Ax, 91/199] 'Il n'emploie jamais la justice sans que nous ne l'ayons préalablement forcé par nos péchés' [lit. "sans/avant nous-ERG forcer..."]

Dans l'extrait suivant, le PG fort est contenu dans l'objet direct (luimême au génitif) du verbe nominalisé :

(11) Iraungitzen da kolera ere [BAT BEDERAK BERE $_{\mathrm{i}}$ falten gogoratzeaz]. [Ax, 210/416]

'La colère s'éteint aussi, chacun se remémorant ses fautes.'

- Passant aux coréférents implicites, on voit qu'il peut s'agir du sujet, comme dans :

(12) Bada fintasunaz, $\left[P R O_{\mathrm{i}} \mathrm{BERE}_{\mathrm{i}}\right.$ hitzaren leial eta iabe izaiteaz], ezta zer esanik. [Ax, 85/187]

'Il n'y a rien à dire concernant le respect de la parole donnée' [litt. "l'être fidèle et maître de son mot"]

(13) Azken erremedioa da $\left[P R O_{\mathrm{i}}\right.$ GEURE $_{\mathrm{i}}$ baitan barrena pensatzea [zein gauza ona den bakean egoitea]]. [Ax, 212/419]

'Le dernier remède est de penser en nous [GÉN] quelle bonne chose c'est que d'être en paix.'

Noter que si, dans (12), on souhaitait donner directement une interprétation générique ou arbitraire à bere, ce type d'explication ne saurait rendre compte de l'occurrence de geure dans (13).

- Mais il peut aussi être un autre argument - ici un objet indirect qui serait au datif s'il était explicite :

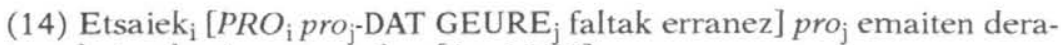
kute okazino ernatzeko. [Ax, 16/66]

'(Nos) ennemis nous donnent l'occasion de nous éveiller en nous [pro-DAT] disant nos fautes.'

4. Voici maintenant quelques extraits montrant la répartition des formes fortes et faibles selon la proximité ou l'éloignement d'un argument coréférent.

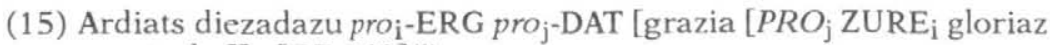
gozatzeko]]. [EZ, 180 $]^{(9)}$

'Obtenez-moi la grâce [de jouir de votre gloire].'

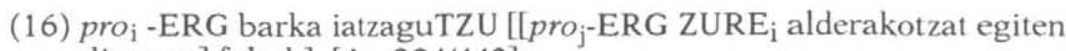
ditugun] faltak]. [Ax, 224/442]

'Pardonnez-nous les fautes que nous faisons à votre encontre.' 
Zure 'votre' n'a d'antécédent local ni en (15) ni en (16), puisque le coréférent est dans la proposition principale ou matrice, et non dans la proposition minimale qui contient le génitif, cf. (4). En (17) par contre, le possessif a un antécent local, le sujet sous-entendu de la nominalisée ; la forme forte est donc de rigueur:

(17) Ardiats diezadazu pro prRG $_{\text {pro }}$-DAT [grazia $\left[\mathrm{PRO}_{\mathrm{j}} \mathrm{NEURE}_{\mathrm{j}}\right.$ hitzen konplitzeko]]. [EZ : 182]

'Obtenez-moi la grâce [de tenir mes promesses].'

Considérons encore l'exemple suivant.

(18) Indazu pro p-ERG pro $_{\mathrm{j}}$-DAT grazia $\left[\mathrm{PRO}_{\mathrm{j}}\right.$ [ENE $_{\mathrm{j}}$ ahalek dirauten] bezanbat ZURE k $_{\mathrm{i}}$ ontzebitzeko]. [EZ : 168]

'Faites-moi la grâce de vous concevoir tant que durent mes forces.'

Les deux génitifs sont faibles. D'une part, en effet, malgré la proximité linéaire du PRO qui coréfère avec ene, et malgré le fait que ce génitif soit dans le SN sujet de sa propre proposition, ce PRO est trop éloigné structuralement du génitif pour que ce dernier soit à la forme forte. D'autre part, zure est également faible : il représente l'objet direct du verbe nominalisé, et son coréférent est également hors de la proposition minimale qui le contient.

Pour finir, notons que lorsqu'un argument est complexe, l'une de ses composantes ne peut servir d'antécédent à un pronom génitif fort :

(19) Prometatu zerauEn gure Jaungoikoak [Abrahani $i_{i}$ eta HAREN $_{\mathrm{i}}$ ondokoei], emanen zerauela Judeako erresuma on hura.

[Ax, 82/182]

'Notre Seigneur promit à Abraham ${ }_{\mathrm{i}}$ et ses $_{\mathrm{i}}$ compagnons qu'il leur donnerait cette bonne terre de Judée.'

Ceci est évidemment dû au fait que si Abrahan est un argument sémantique de 'promettre', il n'en est pas un argument syntaxique. ${ }^{(10)}$

5. Considérons maintenant les cas qui font peut-être difficulté chez Axular. Le seul cas étonnant que j'aie rencontré en proposition conjuguée est le suivant :

(20) Bada mundu haur ezta bertze gauzarik, GURE $\mathrm{i}_{\mathrm{j}}$ heriotzera kondenaturik gaudenon gartzele bat baizen. [Ax, 38/103]

Si la traduction proposée par Villasante : Pues este mundo no es otra cosa que una cárcel en que nosotros estamos condenados a muerte ('Donc ce monde n'est rien d'autre qu'une geôle où nous restons, condamnés à mort'- mais plus littéralement 'où nous sommes condamnés à notre 
mort'), est exacte, on s'attendrait à la présence de la forme forte geure, plutôt qu'à celle de la forme faible gure, étant donné que le PG a, dans cette interprétation, un coréférent dans la proposition minimale qui le contient, le sujet (sous-entendu : $P R O$ ) du verbe egon 'être quelque part, rester'.

Il y a cependant un problème, précisément lié à la forme même de ce verbe: on a bien gauden $O N$, que l'on ne saurait comprendre comme la simple forme relativisée de gaude 'nous sommes/restons'; cette forme relativisée devrait en effet être gauden 'que nous sommes/restons'. La question se pose donc de savoir comment interpréter le suffixe final -on. Il me semble naturel d'y voir un génitif pluriel déictique "proche" répétant gure - en d'autres termes, il paraît souhaitable de traduire l'expression dans son ensemble par: 'Ce monde n'est rien d'autre que notre [gure] geôle, à nous qui sommes [gaudenon] condamnés à mort.'(11)

6. Dans le passage suivant, on attendrait deux fois la forme forte zeure, dans la mesure où la proposition contient un datif (détrimental) pro qui lui coréfère :

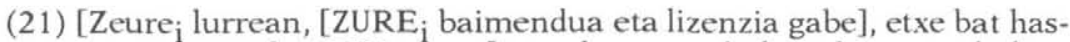
ten pro $_{\mathrm{j}}$ pro $_{\mathrm{i}}$ deraTZUtenean]... ordenatzen du legeak, egotz ahal dezakezula obra berria. [53/127-128]

"Quand on commence à "vous" construire une maison sur votre terre sans votre autorisation ou permission, la loi permet que vous détruisiez la nouvelle construction.'

Pourquoi a-t-on donc la forme faible dans le second cas ? Une hypothèse est que la séquence entre crochets ne soit pas un simple groupe adverbial, mais une proposition non fléchie : dans ce cas elle contiendrait un verbe sous-entendu, du type 'avoir' ou 'recevoir', et donc également un sujet sous-entendu $P R O$ qui serait coindexé avec le sujet indéfini ou générique de $3^{\mathrm{e}}$ personne du pluriel pro ${ }_{j},\left[P R O_{\mathrm{j}} Z U R E_{\mathrm{i}}\right.$ baimendua... IZAN/HARTU gabe] : 'sans $P R O$ (avoir) reçu votre autorisation'. Nous retrouverions alors le cas de figure illustré par les exemples (15) et (16) supra, où la forme du PG est faible parce que la proposition minimale qui contient ce PG ne contient pas d'argument qui lui coréfère.

7. Dans (22), le problème est inversé : pourquoi a-t-on une forme forte alors que le SN possessivé est, apparemment du moins, à l'intérieur d'un constituant de type propositionnel?

(22) DuZUnean pro $_{\mathrm{i}}$ adiskide bat aberatsa, boteretsua eta [ZEURE [Zgi- $_{\mathrm{i}}$ e teko guztietan betiere fin eta leial frogatua]... [84/185-186]

'Quand vous avez un ami riche et puissant qui s'est toujours avéré loyal et fidèle quand vous êtes dans le besoin' [lit. 'dans vos tâches'] 
Deux possibilités s'offrent, qui sont toutefois contradictoires. D'une part, on pourrait avancer l'hypothèse que la séquence entre crochets n'est, finalement, pas de type propositionnel, l'expression zeure egiteko guztietan étant simplement adjointe au syntagme adjectival construit autour de frogatua.

Il me semble cependant préférable d'adopter la solution inverse, parallèle à la proposition faite pour (21): on peut en effet aisément supposer que le verbe implicite de la proposition dont frogatua est le prédicat n'est pas la simple copule izan, mais son correspondant bivalent ou transitif, une forme d'avoir - cf. les paraphrases tout à fait naturelles en basque entre ( $23 \mathrm{a})$ et $(23 \mathrm{~b})$ :

(23) a. (ene) semea eri da '(mon) fils est malade'

b. pro (ene/neure $\left.{ }^{(12)}\right)$ semea eri duT lit. 'j'ai mon fils malade'

En conséquence, au lieu de ( 24 a), qui ferait difficulté du point de vue de la sélection du PG, on pourrait poser (24 b), avec deux arguments phoniquement non-réalisés, un absolutif et un ergatif :

(24) a. [pro ${ }_{\mathrm{i}}$ ZEURE $_{\mathrm{j}}$ egiteko guztietan betiere fin eta leial frogatu $\mathrm{DENa}]$ litt. '(le) qui est fidèle et loyal dans vos affaires'

b. [pro pro $_{\mathrm{i}} \mathrm{ZEURE}_{\mathrm{j}}$ egiteko guztietan betiere fin eta leial frogatu DUZUNa]

litt. '(le) que vous avez fidèle et loyal dans vos affaires'

8. Le cas suivant, repris en (25), est lié à une ambiguïté lexico-grammaticale : doit-on interpréter la forme hedatzea comme un verbe nominalisé, ou comme un nom déverbal?

(25) Hau da ausartzia, presunzionea... eta miseriokordia heltzen den baiño aitzinago ZURE desiraren hedatzea. [99/213-214]

'Ceci est (pure) témérité, et présomption, et une manière d'étendre votre désir au-delà de la portée de la miséricorde divine.'

Si l'on interprète hedatzea comme une forme verbale, 'le fait de s'étendre' (cf. l'anglais extending), on attendrait la forme forte, car le sujet (sous-entendu, mais on a vu plusieurs fois qu'un sujet sousentendu est grammaticalement actif) de ce procès coréfère au PG. Mais les entités en $t(z) e(a)$ sont en fait ambiguës : il peut s'agir également d'un simple nom ('l'extension'), obtenu par dérivation (13); dans ce dernier cas, il n'y a pas de sujet sous-entendu, si bien que la forme faible qui apparaît est alors normale. Or le contexte linguistique immédiat vient corroborer cette alternative : la modification est de type adjectival :... aitzinAGO 'plus avant' et non pas adverbial (on n'a pas : aitzinagoRIK) : la construction semble donc bien totalement régulière. 
9. En ce qui concerne les passages reproduits ci-après par contre, j'avoue n'avoir rien à dire, sauf bien entendu que zeure serait régulier, comme on peut s'en rendre compte aujourd'hui encore en remplaçant la $2^{c}$ personne polie par une troisième personne, et en comparant ensuite le statut respectif de bere et de haren):

(26) Urte honetan gaixto izanik, datorkeienean onduko eta prestutuko zarela diozu. Bada biz hala, zuk diozun bezala, eta gogogan darabillazun bezala gerta dakizun, eta urte hunetan gaixto izanik, ZURE gero horretan, etorkizunean prestu ZAITezin. [101/216]

'Étant méchant cette année, vous dites que vous deviendrez meilleur et vertueux l'année prochaine. Qu'il en soit ainsi, comme vous dites, et que tout se produise comme vous l'imaginez, si bien que, quoique étant mauvais cette année, vous $\mathrm{i}_{\mathrm{i}}$ deveniez vertueux à l'avenir, dans " ce futur vôtre " $^{\text {'. }}$

(27) Non da halaber bitartean, urte honetan [[ZURE gero horretara hel arterainokoan] galtzen duZU] $\mathrm{n}$ denbora ? [ibid.]

'Et pendant ce temps, que dire du temps que vous gâchez cette année (à attendre) de parvenir à ce futur vôtre ?'

En particulier, rien n'indique que la présence d'un démonstratif joue un rôle quelconque ici, mais je reconnais manquer de données à ce sujet. ${ }^{(14)}$

Cependant, dans le cas de (27), il existe peut-être une solution qui rendrait le passage régulier : il est en effet possible d'imaginer que le $P R O$ sujet sous-entendu de hel 'arriver' n'est pas "contrôlé" par le sujet pro de la proposition principale, lui-même marqué par le suffixe -zu (2 pol.), mais par le nom relativisé denbora 'le temps'. ${ }^{(15)}$

10. Dans l'extrait suivant, la forme forte bere n'a apparemment pas d'antécédent local, puisque ikusirik 'voyant' semble n'avoir, outre le SN contenant le PG, qu'un seul autre argument, la question enchâssée [nola proi $_{\text {zihoan ] : }}$

(28) Gizon bat ${ }_{i}$ zihoan behin ... bertze baten hiltzera, eta [ikusirik $\left[B E R E_{\mathrm{i}} \text { adiskide batek }\right]_{\mathrm{j}}\left[\right.$ nola pro $_{\mathrm{i}}$ zihoan]], galdegin zerauKAn... [211/416-417]

'Un fois, un homme $\mathrm{i}_{\mathrm{i}}$ allait en tuer un autre, et, un des ses $\mathrm{s}_{\mathrm{i}}$ amis voyant ce qu'ili allait faire [litt. 'comment il allait'], lui demanda :...'

Noter en particulier que le $P R O$ sujet sous-entendu de ikusirik est contrôlé par bere adiskide batek $k_{j}$, ce qui exclut en principe toute occurrence de la forme forte ${ }^{(16)}$. Il n'est cependant pas impossible de sauver encore une fois les données, en analysant la proposition participiale comme illustrant un cas de prolepse, c'est-à-dire, plus spécifiquement, comme contenant un pro (objet direct du verbe ikusirik) qui fonctionnerait alors bien comme l'antécédent local du pronom fort. 
Il est vrai que la prolepse est généralement condamnée par les basquisants prescriptivistes. J'étaierai donc cette hypothèse avec un exemple tiré d'Axular lui-même :

(29) Orai badirudi EUSKARAK ${ }_{\mathrm{i}}\left[\right.$ pro $_{\mathrm{i}}$ ahalke dela...] [10/55]

'Il semble aujourd'hui que le basque soit intimidé...'

litt. 'Le basque ${ }_{\mathrm{i}}$ semble aujourd'hui qu'il $\mathrm{i}_{\mathrm{i}}$ soit honteux'

11. C'est à un cas plus usuel d'occurrence d'un argument non visible que l'on peut attribuer le tour suivant : ${ }^{(17)}$

(30) Baldin etsaia ${ }_{i}\left[P O_{i}\right.$ GEURE $_{j}$ arimako gazteluan sart:ctik] nahi badugu pro $_{\mathrm{j}}$ begiratu... [256/504]

'Si nous ${ }_{\mathrm{i}}$ voulons empêcher le diable de pénétrer dans le château de

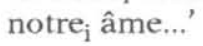

Comme j'ai consacré quelques lignes (cf. les sections 3 et 4) à montrer que toutes les propositions, qu'elles soient conjuguées ou non, d'une part représentent des domaines locaux opaques en labourdin classique, et d'autre part contiennent des arguments éventuellement non-sujets invisibles, il suffit ici de poser que le prédicat sartu $\approx$ sartze$t i k$ a en fait, outre son argument sujet et son argument locatif, un argument datif détrimentaire, ce que tous les dialectes basques de toutes les époques ont toujours permis. Pour nous limiter aux dialectes basques du Nord, il suffira de citer trois traductions de Jean 18, 10, qui font apparaître ce troisième argument dans une proposition conjuguée (31 a-c), puis deux constructions contemporaines dans lesquelles non seulement l'argument détrimentaire proprement dit est silencieux (pro), mais dans lesquelles la forme verbale fléchie qui marquerait le datif de 3 sg. est elle-même absente, (32 a, b) :

(31) Puis Simon-Pierre... frappa le serviteur... et LUI trancha l'oreille droite.

a Simon Pierrisek jo zezan zerbitzaria eta ebaki ziezOn eskuineko beharria. [Liçarrague, 1571]

b ... ebaki ziOen eskuiñeko beharria. [Haraneder, 1742]

c ... moztu ziOen eskuineko beharria. [Duvoisin, 1865]

(32) a ... mutila jo zuen eta eskuineko beharria moztu Ø. [Ezkila, 1974]

b ... mitila jo zian eta esküiñeko beharria moztü $\varnothing$.

[Casenave, 1986, p. 146]

(Voir aussi l'exemple (14) supra, qui est en fait de même type, à ceci près que le pro datif a un coréférent pro rendu plus "visible" par la marque de datif $-k u$ - dans la forme verbale fléchie de la proposition principale. ${ }^{(18)}$ ) 
12. Si les explications qui précèdent (§§ 5 à 11) sont acceptées, seul l'exemple (26) représenterait un contre-exemple absolu aux règles résumées dans la première partie de ce texte. En effet, toutes les éditions consultées présentent zure - mais une coquille n'est pas à exclure, car, dans le dernier cas que j'aie à présenter, c'est la solution la plus naturelle à proposer :

(33) Edirenen duZUE pro ${ }_{\mathrm{i}} \mathrm{ZUEN}_{\mathrm{i}}$ arimentzat bake eta sosegu. [312/616] 'Vous trouverez paix et repos pour votre âme.'

Le PG introduit un circonstant, 'pour votre âme', adjoint à un prédicat dont une $2^{\mathrm{c}}$ personne est argument (le sujet pro de $2^{\mathrm{c}} \mathrm{p}$. pl. représenté par le suffixe-zue dans la forme verbale conjuguée duzue). On s'attendrait donc à la forme forte zeuen plutôt qu'à la faible zuen. Si l'on se reporte maintenant à l'édition fac-similé du texte de 1643, on lit (p. 483) : edirenen duçue CUEN arimentçat..., ce qui est évidemment une erreur : l'orthographe de l'époque exigeait soit une cédille sous le 'c'devant le 'u': ÇUEN (forme faible), soit, pour la forme forte, un 'e' entre le 'c'et le 'u': CEUEN. Face à cette alternative, il suffit donc de corriger en lisant $C E U E N$ plutôt que ÇUEN, ce qui n'est que très naturel une fois que le système d'opposition entre les deux types de formes est pris en compte.

13. En guise de conclusion, il est donc possible de souligner l'extrême régularité de la distribution des formes fortes et faibles de pronoms génitifs chez Axular. Un lecteur (anonyme !) de WSGP m'a écrit, suite à la publication de l'ouvrage dont ce texte constitue le dernier chapitre, pour me dire que le "labourdin classique" tel que je l'y ai décrit était un pur mythe. C'est évidemment une absurdité, mais cela ne signifie pourtant pas qu'il faille en importer de manière prescriptive tous les détails dans la langue moderne unifiée qui est en train de se construire : l'absence de tout rendement fonctionnel de l'opposition entre les deux types de pronoms condamnait nécessairement la belle mécanique axularrienne à disparaître à terme, et l'étude détaillée fournie par WSGP montre que ce système n'a effectivement perduré que très peu de temps.

Georges REBUSCHI

Université Paris III - Sorbonne nouvelle ERS 142 du CNRS 


\section{Sources basques}

[Ax] Axular, Pedro de. 1643. Guero. Bordeaux : Milanges. Ed. avec trad. espagnole par L. Villasante, Barcelone : Juan Flors, 1964. Ed. fac-sim. de la première, Euskaltzaindia, Bilbao, 1988.

Casenave, Junes. 1986. Egün oroetako irakurgeiak. Zarauz : Itxaropena.

Detchepare, Bernard. 1545. Lingvae Vasconvm Primitice. $\mathrm{N}^{\text {tles }}$ éd. : (i) par L. Aquesolo, R. Lafon, \& L. Michelena, avec trac. esp. et fr., Saint-Sébastien : Edili, 1968; (ii) par P. Altuna, Bilbao: Mensajero, 1980.

Duvoisin, Jean. 1865. Bible edo Testament Zahar eta Berria [...]. Londres, 1859-65. Fac-sim., Bilbao : Gran Enciclopedia Vasca, 1972.

[EZ] Etcheverry Joannes, de Ciboure. 1627. Manval devotionezcoa [...] $2^{e}$ éd., Bordeaux, MongironMillanges (2 vols.), 1669. Fac-sim. de cette dernière en 1 vol., SaintSébastien : Hordago, $1979^{(19)}$.

"Ezkila". 1974. Jesu Kristoren Berri Ona. Belloc : Éditions Ezkila.

Haraneder, Joannes de. 1742. Jesu Christoren Evangelio Saindua. Ms. éd. par P. Altuna, Bilbao : Euskaltzaindia, 1990.

Liçarrague, Joannes. 1771. Iesus Christ Gure Iaunaren Testamentu Berria - Kalendera - $A B C$ edo Christinoen Instructionea. La Rochelle: Haution. Réimpr. en facsim., avec une introduction par Th. Linschmann \& H. Schuchardt, Strasbourg : Trübner, 1900. Facsim. de cette réimpr., Bilbao : Euskaltzaindia, 1990.

\section{Références linguistiques}

Lafitte, Pierre. 1962. Grammaire basque; dialecte navarro-labourdin littéraire. Bayonne: Amis du Musée Basque \& Ikas.

Rebuschi, G. 1989. "Is there a VP in Basque?". In P. Muysken \& L. K. Marácz (éds.), Configurationality : the Typology of Asymmetries (Dordrecht : Foris), 85-116.

Rebuschi, G. 1995. "Weak and Strong Genitive Pronouns in Northern Basque: A Diachronic Perspective". In J. I. Hualde, J. A. Lakarra \& R. L. Trask (éds.), Towards a History of the Basque Language (Amsterdam, Benjamins), 313-356.

Reinhart, T., \& E. Reuland. 1993. "Reflexivity" - Linguistic Inquiry 24/4 : 657-720.

Sarasola, Ibon. 1980. "Nire - neure, zure-zeure literatur tradizioan". Euskera 25/2 : 430-446.

Trask, Robert L. 1995. "On the History of the Non-Finite Verb Forms in Basque". In J. I. Hualde, J. A. Lakarra \& R. L. Trask (éds.), Towards a History of the Basque Language (Amsterdam, Benjamins), 207-234. 


\section{NOTES}

1. La raison pour laquelle je ne les appelle pas "neutres" et "emphatiques" apparaîtra dans le texte ; en deux mots, il n'y a emphase que s'il y a choix pour le locuteur, ou, à la rigueur, si le contexte force (parce qu'il apporte un élément contrastif) à employer un élément marqué comme focal ; or, on le verra, rien ne permet de dire que, en labourdin classique, les formes que j'appelle "fortes" étaient emphatiques - au contraire, leur distribution était entièrement régie par des contraintes structurales, c'est-à-dire purement mécaniques et aveugles, et ce, tant pour les deux premières personnes (du singulier comme du pluriel) que pour la troisième.

2. Le nombre qui précède les deux points renvoie à la page de l'édition de P. Altuna (1980) et celui qui les suit, au numéro du vers dans cette même édition ; la traduction est par contre de R. Lafon.

3. Le problème technique est le suivant : on admet que la proposition subordonnée a un sujet sous-entendu qui joue un rôle syntaxique, au moins en ( 2 a) : la forme génitive de l'objet "direct" y est donc régulière ; en ( 2 b) par contre, si la construction basque était celle qu'indique la traduction de Lafon, on attendrait la forme réfléchie neure buruaren... (Faut-il préciser que j'appelle ici "directs" des compléments effectivement au génitif, tout simplement parce qu'ils seraient à l'absolutif si la proposition était fléchie ?).

4. En labourdin, les pronoms intensifs (dont zeuron) sont exclus de tels contextes, exactement de même que les génitifs faibles, et ce, pour des raisons qui apparaîtront plus loin ; la question est donc de savoir ce qui rend le pronom intensif possible ici.

5. Durant la seconde moitié du XVII' siècle, le système très régulier décrit ci-après s'est désintégré, et de plus extrêmement rapidement - cf. WSGP, section 3.

6. Cf. (3) ; je laisse de côté la question des objets directs de verbes nominalisés comme en ( $2 \mathrm{a})$; voir cependant la note 14 à ce sujet. Deux autres cas ne seront pas abordés : (i) celui des formes fortes dans les SN vocatifs (cas traité en partie dans WSGP, §5.3, car la description qu'en propose Sarasola (1980) ne me paraît pas satisfaisante), et celui des prédicats secondaires (ce que la grammaire traditionnelle appelle "constructions avec attribut de l'objet"), lorsqu'un pronom génitif est dans ce prédicat, et que son coréférent n'est précisément pas l'objet direct, mais le sujet de la proposition globale. Les données dont je dispose sur ce cas de figure me semblent contradictoires.

7. Les références à Axular [Ax] indiquent d'abord la pagination de l'édition de 1964, puis la section selon la numérotation de L. Villasante.

8. Il n'y a donc pas d'effets dits de croisement faible (angl. Weak Cross-Over Effects), cf. Rebuschi (1989).

9. "EZ" : Etcheverry de Ciboure ; les renvois sont tous aux pages du $2^{\circ}$ vol. du Manval devotionezcoa, renumérotées à partir de 1 dans l'édition fac-sim. employée (cf. la bibliographie).

10. Cf. Lafitte (1962: 93) qui dit en terme intuitifs que, dans le telles constructions, "le verbe déborde" le sous-argument antécédent du possessif. Pour un point de vue théorique moderne sur ces questions, voir Reinhart et Reuland (1993).

11. Cette interprétation présuppose que deux autres faits soient avérés - ce qui est le cas : (a) que les expressions en apposition dupliquent le cas des éléments auquels elles sont apposées, et (b) que les adverbiaux (en particulier de lieu) puissent rester invisibles.

12. Le possessif est inutile, le possesseur étant exprimé par l'argument ergatif marqué par le suffixe - $t$. Ajoutons que dans la langue spontanée d'aujourd'hui, le PG fort neure a disparu (de même que les autres PG forts de $1^{\text {rt }}$ et $2^{\text {e }}$ personnes).

13. Voir Trask (1995) pour une étude détaillée de cette opposition. 
14. Le rôle de facteur opacifiant (dans les situations syntaxiques où la localité est décisive) joué en anglais, mais en français aussi, par les démonstratifs est bien connu; il est illustré par la différence d'acceptabilité entre les deux phrases suivantes :

(i) Nous avons acheté DES photos les uns des autres

(ii) *Nous avons acheté CES photos les uns des autres

15. Il est également exclu d'envisager que zure soit ici un sujet génitif : dans ce cas, on attendrait à nouveau zeure - comparer (2b), où un PG sujet est fort car coréférant à un argument de la proposition principale.

16. Ajoutons que l'ordre des constituants interdit de considérer que le pro datif marqué par le suffixe-ka- de la proposition radicale soit l'élément qui rende bere licite : il faudrait en effet admettre que le SN entier bere adiskide batek soit lui-même le sujet de galdegin zerauKAn '(il) lui demanda', tout en se trouvant inséré entre ikusirik et la complétive en nola... - alors que ce $\mathrm{SN}$ ne peut que coréférer à ce nouveau sujet invisible pro.

17. La solution à ce contre-exemple apparent m'a été suggérée par R. L. Trask (p.c.).

18. Balayer d'un coup de plume les ex. (32) comme relevant de la "simple" ellipse ne peut suffire à mes yeux, dans la mesure où l'ellipse ne peut se reconnaître que moyennant la récupérabilité morpho-syntaxique des éléments effacés. Or ce qui est effacé ici, c'est ou bien la forme même de l'auxiliaire zuen, ou bien son contenu grammatical - mais dans les deux cas, il n'y a d'information que sur la personne et le nombre du sujet ( $3 \mathrm{sg}$.) et de l'objet (id.), et aucune information concernant un éventuel $3^{\prime}$ argument au datif...

19. Les pages citées de cet auteur (ex. (15), (17), (18)) sont en fait celles du $2^{\epsilon}$ volume, réimprimé sans renumérotation à la suite du premier. 